\title{
An Effective Diagnosis of Diabetic Retinopathy with Aid of Soft Computing Approaches
}

\author{
Nasr Y. Gharaibeh ${ }^{1}$ and Abdullah A. Alshorman ${ }^{2}$ \\ 1. Electrical Engineering Department, Al-Balqa Applied University, Al-Huson University Colleg, Al-Huson 21510, Jordan \\ 2. Mechanical Engineering Department, Al-Balqa Applied University, Al-Huson University Colleg, Al-Huson 21510, Jordan
}

Received: June 07, 2016 / Accepted: June 16, 2016 / Published: August 31, 2016.

\begin{abstract}
DR (diabetic retinopathy) is a most probable reason of blindness in adults, but the only remedy or escape from blindness is that we have to detect DR as early. Several automated screening techniques are used to detect individual lesions in the retina. Still it takes more dependency of time and experts. To overcome those problems and also automatically detect DR in easier and faster way, we took into soft computing approaches in our proposed work. Our proposed work will discuss several amounts of soft computing algorithms, it can detect DR features (landmark and retinal lesions) in an easy manner. Processes includes are: (1) Pre-processing; (2) Optic disc localization and segmentation; (3) Localization of fovea; (4) Blood vessel segmentation; (5) Feature extraction; (6) Feature selection; Finally (7) detection of diabetic retinopathy stages (mild, moderate, severe and PDR). Our experimental results based on Matlab simulation and it takes databases of STARE and DRIVE. Proposed effective soft computing approaches should improve the sensitivity, specificity and accuracy.
\end{abstract}

Key words: Diabetic retinopathy, soft computing, microaneurysm, exudates, hemorrhage and blood vessel.

\section{Introduction}

DR (diabetic retinopathy) is a leading problem of adult people. In this world, nearly 93 million people could be affected by DR. People who would be affected by DR, their age come nearly 40+. Most of the Americans (7.7 millions) had been affected by DR [1]. Usually, DR has two stages such as NPDR (non-proliferative diabetic retinopathy) and PDR (proliferative diabetic retinopathy). NPDR has no symptoms, it can detect only by fundus photography. Microaneurysms come under NPDR. Another stage is PDR, which has the components (features) of neovascularization and vitereos hemorrhage.

Blindness is the major problem in today's world. So, the various stages of DR can be analyzed and examined by retinal fundus images (color images). Some image

Corresponding author: Abdullah A. Alshorman, Ph.D., associate professor, research fields: bio-fluid dynamics, energy conversion and utilizing, renewable energy and thermofluid simulation and modeling. analysis tools [2] are used for detection of DR stages and features.

Diabetic NPDR stages are classified into mild, moderate and severe [3].

(1) Mild NPDR: This kind of NPDR is also called as MA (microaneurysm), which is small swellings in small blood vessels in the retina.

(2) Moderate NPDR: This is mainly progress based on blood vessels which nourish the retina to be blocked.

(3) Severe NPDR: In this stage, large amount of blood vessels are blocked and arrest blood supply into many retinal areas. This could cause lack of oxygen. Problems of these should raise blot hemorrhages, bleeding in the veins and also intra retinal micro vascular abnormalities.

(4) PDR: In PDR [4], fluids are leakage in large amount and it could lead to seriousness. Generation of bleeding is caused by some or high pressure in the blood vessels. That kind of bleeding is also called as hemorrhage. Vision loss and scarred retina come 


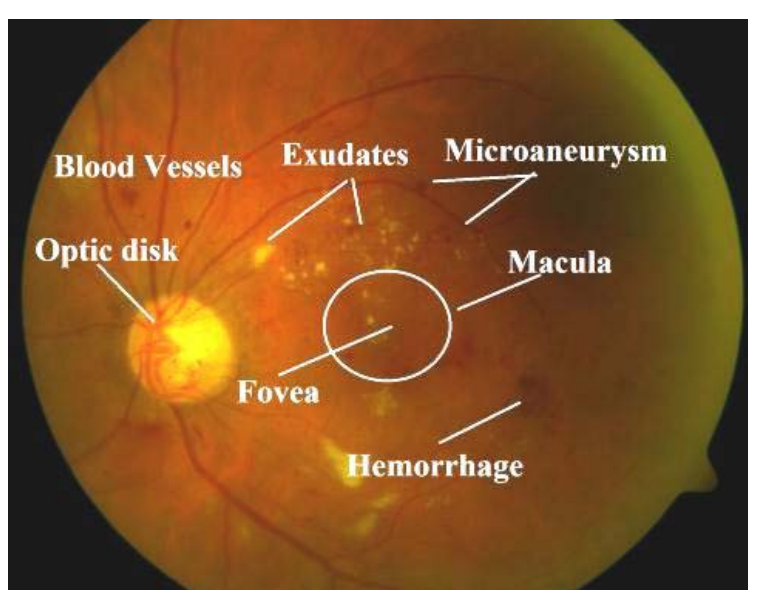

Fig. 1 DR related pathologies and retinal main regions.

with the reason of hemorrhage. Fig. 1 shows the DR pathologies and retinal main regions.

Basic symptoms of DR are:

(1) Rings or black spot;

(2) Blurred vision;

(3) Corner eyes (vision) are affected;

(4) Pressure (or) pain in eyes.

Retinal features can be classified into two types. There are landmark features and retinal lesions. Landmark features are known as optic disc, blood vessels and fovea. Retinal lesions are called as microaneurysm, hemorrhage and exudates. Both features are appearing slightly similar. Similarities are:

(1) Blood vessel, hemorrhage and microaneurysm are in same color. Larger hemorrhage differs only its geometrical features. Smaller hemorrhage is same as in color, contrast and geometry.

(2) Optic disc and exudates show similar as in color, exudates are bright region.

(3) Microaneurysm, hemorrhage and fovea look like the same in its color but fovea has a dark region.

Soft computing approaches $[5,6]$ are mostly focused on automatically detection, segmentation and classification process. Soft computing approaches are very commonly encouraged for medical images. Medical images are generally segmented and classified for detection and diagnosis of disease. Fig. 2 depicts the soft computing approach process.

Contribution of our proposed work as follows:

(1) Retinal blood vessel segmentation based on PFCM (possibilistic fuzzy C-means);

(2) Feature extraction to be done by the combination of wavelet and co-occurrence matrix;

(3) Feature selection process to be taken by ANFIS (adaptive neuro fuzzy inference system);

(4) Classification of normal and abnormal categories is done with the help of SVM (support vector machines) and also detection of severity of DR such as mild, moderate, sever and PDR will be done by type-2 fuzzy logic system.

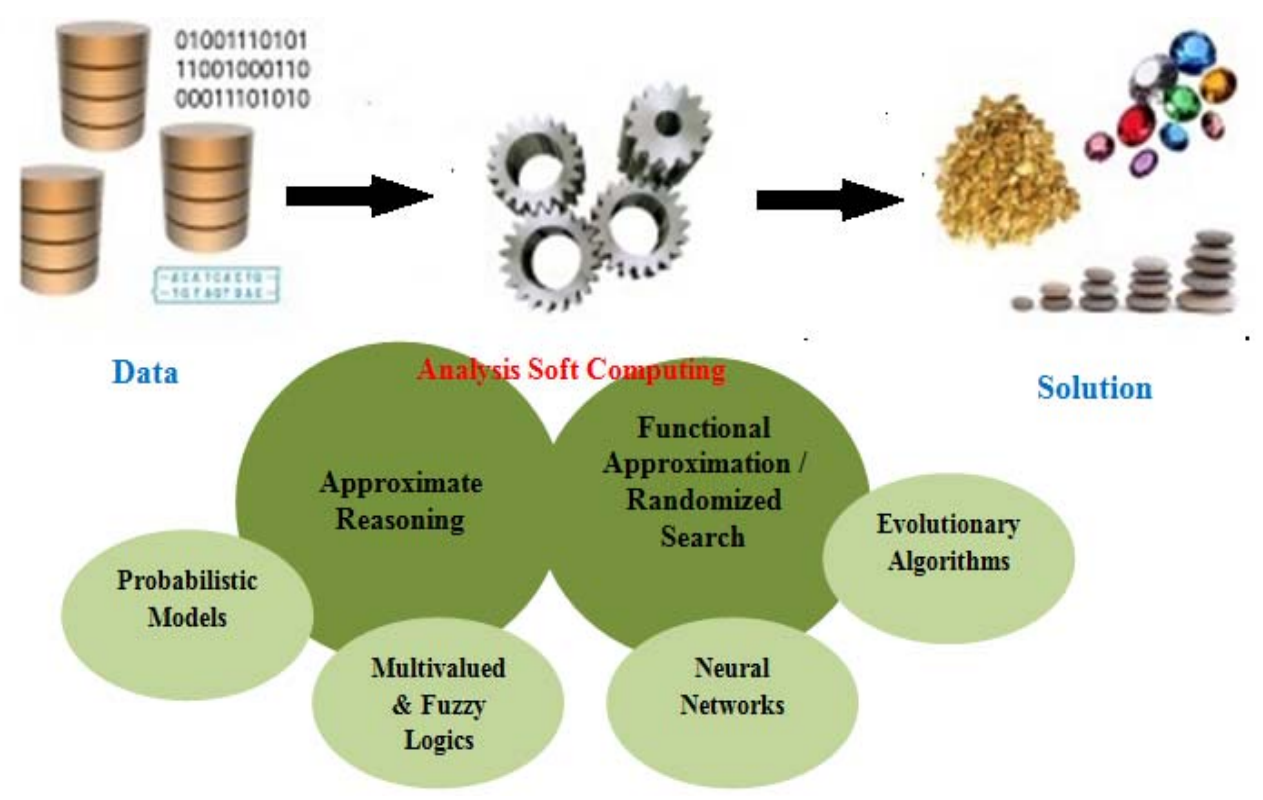

Fig. 2 Soft computing approach process. 
The remaining sections of this work are as follows. Section 2 explained the details of related work and proposed overview is presented in Section 3. Section 4 talked about experimental setup and results. Finally Section 5 concluded the overall proposed concept.

\section{Related Work}

Retinal diseases are discussed in several papers. Diabetic retinopathy disease is mostly identified by retinal fundus images. In Refs. [14, 15, 17] clearly explained about diabetic retinopathy. This includes the processes of pre-processing, optic disc localization and segmentation, segmentation of retinal vasculature, localization of fovea and macula then detection of DR. Several methods are discussed and thrashed out for detection of diabetic retinopathy. Here, classification process only identifies the normal and abnormal retinal images. Performance metrics of sensitivity, specificity and accuracy increased according to the performance of classification and feature selection methodologies. Severity of diabetic retinopathy could be thrashed out in Refs. [29, 30]. Usually, stages of DR are classified as mild, moderate, sever and PDR. Classifiers used in that were achieved below $91 \%$ of accuracy. Exudates are an important retinal lesion which could be automatically detected in Ref. [7].

For detection of exudates, we just take the green channel of retinal image for adjustment of intensity values. Here, separation of true and false exudates should be more importantly spoken. Local binary patterns are well known method for screening retinal disease explained in Ref. [8]. Automatic segmentation of blood vessels is in Refs. [10, 11]. Curvature based algorithm had classification of vessels as opposed to vessel based measures, simplicity of measure and low computation burden. Segmentation blood vessels should include the databases of DRIVE, STARE and DIARETDBI. Optic disc boundary detection and vessel origin can be segmented in Ref. [9]. Here, we extracted the bright regions and classified OD (optic disc) and Non-OD. For classification 6 region features are extracted and it could be done by Gaussian mixture model. Automatic detection of Microaneurysm could be explained in Refs. [12, 22]. Methods involved in that were rotating cross section profile analysis and first order statistics. Those methods are not detecting the other features in the retinal lesions.

Hemorrhage detection is an important part in a detection of diabetic retinopathy. Hemorrhage classification was done by neural network classification. Here, detection of hemorrhage includes region growing and some inverse method. Detection of hemorrhages was hard to distinguish from blood vessels, microaneurysm and fovea, because it looks like low contrast. In this case we need additional effective detection of hemorrhage methodology needed. In Ref. [22] hemorrhage detection was based on features. Here, we performed splat-wise features and pixel based feature responses. Classification could be done with the help of three steps such as initial feature selection or filtering, K-nearest neighbor classification and then perform post-processing. But this kind of screening system reaches the ROC (receiver operating characteristic) curve up to 0.96 .

\section{Proposed System Overview}

Proposed system should encourage detection of DR in an effective manner. For that purpose we have to introduce soft computing algorithms or approaches for the processes of segmentation and classification. Our proposed system steps consist of (1) Pre-Processing the retinal fundus color images; (2) Optic disc localization and segmentation based on intensity variation and snake algorithm; (3) Localization of fovea; (4) Retinal blood vessel segmentation done with the help of PFCM; (5) Feature extraction process to be done based on the combination of wavelet and co-occurrence matrix; (6) ANFIS is a type neuro and fuzzy system and it can select the features in a proper manner; (7) Finally, detection of DR stages (mild, moderate, severe and PDR) is classified by type-2 fuzzy logic system. The overall architecture of our proposed system can be 


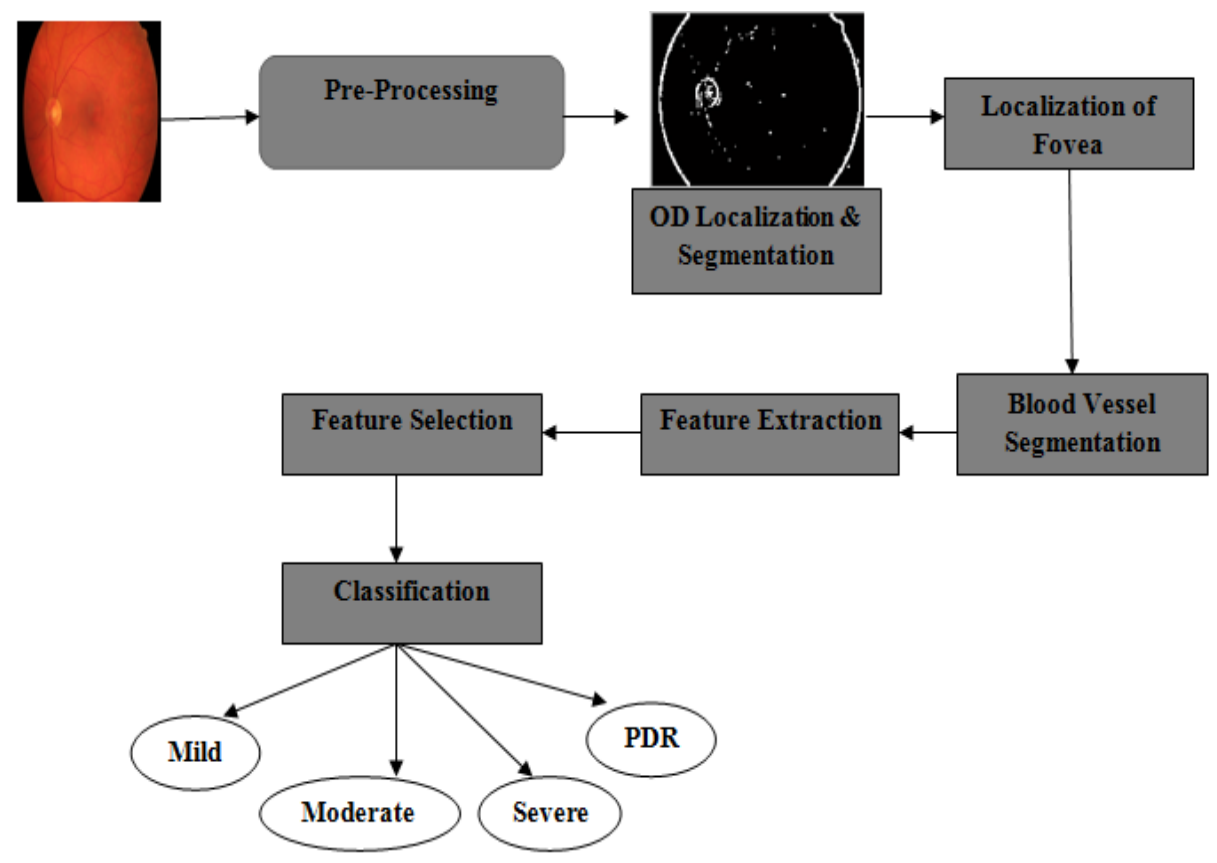

Fig. 3 Architecture of proposed system.

depicted in Fig. 3.

\subsection{Pre-processing}

Pre-processing is an important step in all medical image processing methods. We only used pre-processed image for further progress. This pre-processing step has three stages such as

- Intensity conversion;

- Denoising;

- Contrast enhancement.

In intensity conversion we first take the RGB (red, green and blue) input image which has RGB channels. Its green channel can emit best contrast among vessels and the background. The reason why not choosing other channels is that they are too noisy. Green channel used to convert an intensity of an image [4].

Denoising is mainly focused to neglect noises present in the fundus images. For that we have to use modified spatial median filtering [16]. This modified spatial median filter can easily identify the originality of the center point. Here, each point within the mask is to be computed after finding spatial depths, which could help us to decide if the mask center point is an original one. Replacement of current representative point can be applicable. Similar point of the set is elected by having smallest spatial depth point. That ranking plays an important role for back forwarding the most corrupted points.

Contrast enhancement is a very important thing for associating uneven illumination in the fundus image. Here, we use adaptive histogram equalization method for improving the quality of an image. This technique can easily alter the dynamic rabge, it provides the result of altered contrast of an image.

\subsection{Optic Disc Localization \& Segmentation}

Optic disc localization is commonly used to mask the approximate center within a specific region. Because most of the distractors like exudates, blood vessels are looked like the same. So we have placed the boundary to an outer area of an optic disc for purpose [17].

After OD (optic disc) localization, we have to move to the process of OD segmentation. This segmentation process will be done with the help of snake algorithm gradient vector flow which could easily fit an edge of the OD. This kind of OD segmentation should improve the performance of optic disc segmentation [18]. 


\subsection{Localization of Fovea}

In human vision, fovea is an important part, because the dedicated cones of fovea can destroy human eyes to become blind. Fovea and microaneurysm, hemorrhage can look same in color. So we must localize the fovea for easy detection of DR. After OD localization \& segmentation fovea need to be localize. For localize fovea region [19], we have to take image I, which contain more blood vessels. $\mathrm{C}$ is the exact center of $\mathrm{OD}$. Normally, horizontal line passing through the center which has a distance of $2.5 \times d$ in a direction of centroid. In order to extract fovea region a strip of width $k$ pixels are selected through the horizontal line. Sliding window mask is applied to $k \times k$ pixels, which starts from point in upward and downward window. Then calculate number of black pixels lying in the window. At last, maximum run length of zeros in the chain enables to easily localize the fovea region.

\subsection{Retinal Blood Vessel Segmentation}

Blood vessel segmentation is used to get only the blood vessel with black background. This vessel segmentation process is to be done with the datasets of STARE and DRIVE. PFCM (possibilistic fuzzy C-means) algorithm should ensure the blood vessel segmentation.

PFCM segmentation process should be mentioned below:

Objective function of optimization problem can be stated below,

$$
\begin{aligned}
J_{m, \eta}(U, T, V ; X) & =\sum_{i=1}^{c} \sum_{k=1}^{n}\left(a u_{i k}^{m}+b t_{i k}^{\eta}\right) D_{i k A} \\
& +\sum_{i=1}^{c} \gamma_{i} \sum_{k=1}^{n}\left(1-t_{i k}\right)^{\eta}
\end{aligned}
$$

where,

$$
\begin{gathered}
\sum_{i=1}^{c} u_{i k}=1, \forall k \\
0 \leq u_{i k} \leq 1,0 \leq t_{i k} \leq 1, \\
a>0, b>0, \gamma_{i}>0, m>1, n>1
\end{gathered}
$$

Above mentioned constraints are defined by the users, and $u_{i k}$ is a relative importance of fuzzy membership, $J_{m, \eta}$ is an objective function, $U$ is a partition matrix, $T$ is a typicality matrix, $V$ is a vector of cluster centers, $X$ is a set of all data points, $x$ represents the data points, $n$ is the number of data points and $c$ is the number of cluster centers, these all are described by $\mathrm{S}$ coordinates.

$D_{i k A}$ also represented by, $D_{i k A}=\left\|X_{k}-V_{i}\right\| A$ is an any norm which can be used to calculate the distance between cluster centers of $i$ and $k$. This distance could be calculated using the form of euclidean distance formula presented below,

$$
D_{i k A}=\left[\sum_{j=1}^{s}\left(x_{k j}-v_{i j}\right)^{2}\right]^{1 / 2}
$$

Relatively importance of the typicality value and fuzzy membership value presented in the objective function can have the constants of $a$ and $b$.

The PFCM should use the objective function of PCM and FCM. This could be symbolized below Eqs. (3) and (4).

$$
\begin{aligned}
\min \left\{J_{m}(U, V ; X)\right. & \left.=\sum_{k=1}^{n} \sum_{i=1}^{c} u_{i k}^{m}\left\|x_{k}-v_{i}\right\|^{2} A\right\} \\
\min \left\{P_{m}(T, V ; X, \gamma)\right. & \\
& =\sum_{k=1}^{n} \sum_{i=1}^{c}\left(t_{i k}\right)^{m}\left\|x_{k}-v_{i}\right\|^{2} A \\
& \left.+\sum_{i=1}^{c} \gamma_{i} \sum_{k=1}^{n}\left(1-t_{i k}\right)^{m}\right\}
\end{aligned}
$$

We have to overcome the scaling problem by giving higher values to the typicality and membership alternatively which will reduce the outliers. Scaling optimization problem is also reduced and it is applicable for large datasets. According to the above mentioned process retinal blood vessels are segmented properly. 


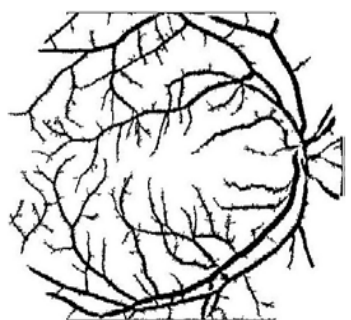

(a)

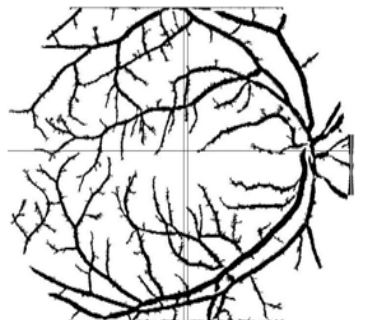

(b)
Fig. 4 Localization of fovea (a) blood vessels of image; (b) horizontal line and vertical strip.

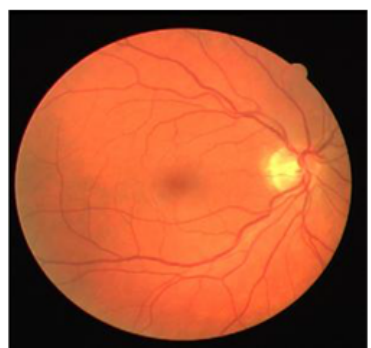

(a)

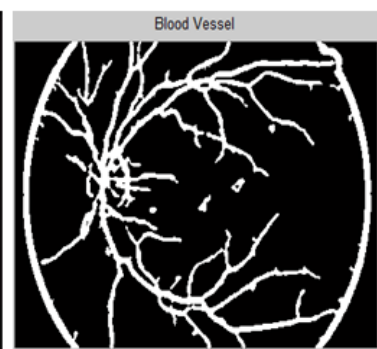

(b)
Fig. 5 Blood vessel segmentation (a) input image; (b) blood vessel segmentation.

\subsection{Feature Extraction}

Feature extraction is the most important task in DR. Normally, features are classified into landmark features and retinal lesions. Already we have to localize and segment the landmark features. Here, we have to extract the retinal lesions like Microaneurysm, Hemorrhage and Exudates. For feature extraction we are going to apply wavelet $\&$ co-occurrence matrix. At first, decompose the fundus image as in ero level and then it could be proceeded by co-occurrence matrix. Decomposition can be depicted in Fig. 6 .

Co-occurrence matrix procedure is followed below:

Formulation of co-occurrence matrix makes use of orientation of angle $\theta$ which occurs between the pair of gray levels and the axis. We consider four directions, they are $\theta=0^{\circ}, 45^{\circ}, 90^{\circ}, 135^{\circ}$. The corresponding gray-level co-occurrence matrix is given as,

where,

$$
p\left(i, j \mid d, 0^{\circ}\right)=\left\{\left(x_{1}, y_{1}\right),\left(x_{2}, y_{2}\right)\right\}
$$

$$
\begin{gathered}
i=I\left(x_{1}, y_{1}\right), j=I\left(x_{2} y_{2}\right),\left|x_{1}-x_{2}\right|=0^{\circ}, \\
\left|y_{1}-y_{2}\right|=d
\end{gathered}
$$

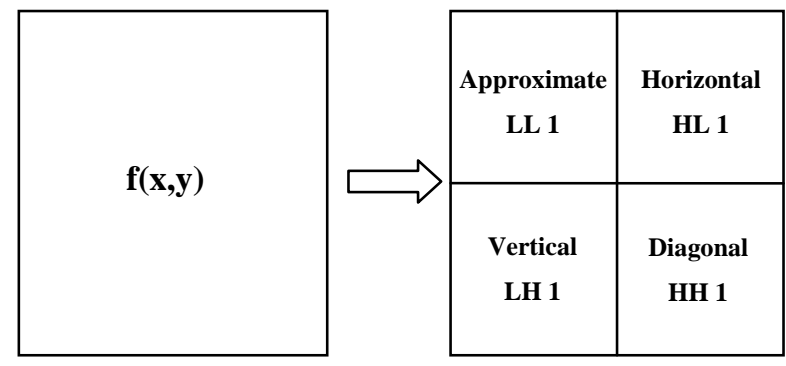

Fig. 6 Decomposition level.

The above mentioned equation is reconstructed by modifying $\theta$ orientation for the remaining three angles. Next, the gray level co-occurrence is defined as $p(i, j \mid d, \theta)$ with respect to the distance $d$ and angle $\theta$. Further the probability value of gray level co-occurrence matrix is given as,

$$
\mathrm{p}(\mathrm{i}, \mathrm{j} \mid \mathrm{d}, \theta)=\frac{p(i, j \mid d, \theta)}{\sum_{i=1}^{256} \sum_{j=1}^{256} p(i, j \mid d, \theta)}
$$

From the estimated $p(i, j \mid d, \theta)$ component, we get features which can be further utilized for extraction process. The texture features are calculated using,

(1) ASM (Angular second moment)

It measures the number of repeated pairs. Only limited number of gray levels is presented in the homogeneous scene. ASM can be calculated as follow,

$$
\mathrm{ASM}=\sum_{i}^{U} P^{2}[i . j]
$$

\section{(2) $\mathrm{CN}$ (Contrast)}

Local intensity variation of an image and it will favor contributions from $P(i, j)$ away from the diagonal, i.e. $\mathrm{i} \neq \mathrm{j}$. Contrast can be calculated using the equation,

$$
\text { Contrast }=\sum_{i}^{U} \sum_{j}^{V}(i-j)^{2} P[i, j]
$$

(3) ET (Entropy)

This parameter calculates the randomness of gray-level distribution.

$$
\text { Entropy }=-\sum_{i}^{U} \sum_{j}^{V} P[i, j] \log P[i, j]
$$

(4) CR (Correlation)

It provides the correlation between two pixels 
presented in the pixel pair.

$$
\text { Correlation }=\sum_{i}^{U} \sum_{j}^{V} \frac{(i-\mu)(j-\mu) P[i, j]}{\sigma^{2}}
$$

where, $\mu$ and $\sigma$ indicate mean and standard deviation.

(5) MN (Mean)

Parameter is used to calculate the mean of gray level in an image. It can be manipulated as,

$$
\text { Mean }=\frac{1}{2} \sum_{i}^{U} \sum_{j}^{V}(i P[i, j]+j P[i, j])
$$

\section{(6) $\mathrm{VN}$ (Variance)}

Variance is used to explain the overall distribution of gray level. The following equation is used to calculate the variance,

$$
\begin{array}{r}
\text { Variance }=\frac{1}{2} \sum_{i}^{U} \sum_{j}^{V}\left((i-\mu)^{2} p[i, j]\right. \\
\left.+(j-\mu)^{2} P[i, j]\right)
\end{array}
$$

\subsection{Feature Selection}

Feature selection process will help us to reduce the feature we had taken and also extracted from retinal images. Above features (textures) are used for detection of DR. This feature selection process can select features in most relevant and informative. ANFIS based feature selection process is included in our proposed work for easy detection of DR. Steps are in Ref. [25].

ANFIS is mainly focusing the optimization of the fitness function. The proposed subset feature selection process can be described as follows,

Step1: Extract number of features from GLCM (gray level co-occurrence matrix) process and denoted as feature 1 , feature 2 , etc.

Step 2: Parameters to be initialized for genetic algorithm population size $=10$ and maximum chromosome length $=7$.

Step 3: From the possible solution subspace randomly selected an initial subset of features.

Step 4: Features are represented by binary values.
Binary values 0 and 1 are used for presence and absence of features.

Step 5: Fitness function determined by ANFIS.

$$
\begin{array}{r}
\text { Fitness (f) }=\sum_{i=1}^{20} \frac{T P}{T P+F N}+\frac{T N}{T N+F P} \\
+\frac{T P+F N}{T P+T N+F P+F N}
\end{array}
$$

Here, TP, TN, FP and FN values are calculated already mentioned in Ref. [25].

Step 6: Maximum threshold value for a feature subset represented by Fitness max $_{\text {. }}$.

Best Feature Subset $=$ Fitness $(f)-$ Fitness $_{\max }$

\subsection{Detection of $D R$}

In this paper, type-2 fuzzy logic system is used to classify DR as mild, moderate, severe and PDR. Before classifying stages of DR we have to separate normal and abnormal retinal images by using SVM (support vector machine) in Ref. [15]. After that we have to detect DR stages. Type-2 fuzzy logic system processed in Refs. [26, 27] can be followed here.

The following equation based T2FLS can be formulated,

$$
\begin{aligned}
& \overline{\bar{A}}=\int_{x \in X} \frac{\mu_{\bar{A}(x)}}{x}=\int_{x \in X} \frac{\left[\int_{x \in X} \frac{f_{x}(u)}{u}\right]}{X J_{x}}, X J_{x} \leq[0,1] \\
& f_{x}(u) \rightarrow \text { Blurred membership function } \\
& J_{x} \rightarrow \text { Original membership }
\end{aligned}
$$

A region between the blurred membership function is also called an FOU (footprint of uncertainty) [28]. This can be expressed as follows,

$$
\begin{aligned}
F O U(\overline{\bar{A}})= & U_{\forall x \in X} J_{x} \\
& =\left\{(x, u): u \in J_{x} \leq[0,1]\right\}(23) \\
\overline{\mu_{\bar{A}}} & =\overline{F O U}(\overline{\bar{A}}) \& \underline{\mu_{\bar{A}}}=\underline{F O U}(\overline{\bar{A}})
\end{aligned}
$$

FOU can be constructed from upper membership function and the lower membership function.

$0 \leq \mu_{\bar{A}}(x, u) \leq 1$, called as secondary membership function. In T2FLS the secondary membership function is calculated by Gaussian interval. The Gaussian 
interval type selecting secondary membership function is described below,

$$
\mu_{\bar{A}}(x, u)=1 \forall_{u} \in J_{x} \leq[0,1]
$$

Next, we move onto the general architecture of fuzzy logic system. This system can be performed and frequently tune the lower and upper bound parameter of FOU. All the parameters of the MF (membership function) are incorporated into structure of chromosome. Then we are involving the genetic algorithm for further classification process. In that we have to apply the fuzzy type based on Mamdani rule.

$$
\begin{gathered}
R_{i} \text { : If } x_{1} \text { is } \tilde{F}_{i 1}, x_{2} \text { is } \tilde{F}_{i 2} \text { and ... } x_{n} \text { is } \tilde{F}_{i n} \\
\text { Then } y \text { is } \tilde{G}_{i}
\end{gathered}
$$

where, $i=1, \ldots, \mathrm{M}$ is the number of rules, $\mathrm{X}=\left(x_{1}, x_{2}, \ldots\right.$, $x_{n}$ ) is a given sample, $\widetilde{F}_{i 1}, \widetilde{F}_{i 2}, \ldots, \widetilde{F}_{i n}$ are antecedent T2FLS and $G_{i}$ is a consequent T2FLS.

\section{Experiments and Results}

Our proposed soft computing approaches should be implemented by using Matlab simulation. In detection of DR we introduce soft computing approaches at the stages of blood vessel segmentation, feature selection and also classification. Our results are compared with previous classifier with the databases of STARE and DRIVE.

\subsection{Database}

In our experiments we use two kinds of databases for performing detection DR task. Detailed explanation about these databases is mentioned below.

\subsubsection{STARE}

STARE database was launched by Michael Goldbaum, MD, University of California and also funded by the national institutes of health. Clinical images were produced, it contains 20 images for blood vessel segmentation and some slides contain pathology. Well, observers could manually segment all the images.

\subsubsection{DRIVE}

DRIVE database has been established for performing comparative analysis on segmentation of blood vessels in retinal images. This publicly available database shall be consisted of 40 color fundus photographs totally. These images are acquired from CCD (charge-coupled device) camera with 8 bits per color plane at $768 \times 584$ pixels.

\subsection{Performance Metrics}

Performance metrics must be taken for measuring performance of our proposed system and also compared with state-of the art techniques. Most frequently used metrics are such as sensitivity, specificity and accuracy.

Sensitivity:

Sensitivity can be measured by the proportion of positives, disease affected people can be correctly identified. This can be represented by

Sensitivity $(\%)=\mathrm{TP} / \mathrm{TP}+\mathrm{FN} \times 100 \%$

Specificity:

Specificity shall be measured by the proportion of negatives, people who could not be affected are correctly identified. This can be represented by

Specificity $(\%)=\mathrm{TN} / \mathrm{TN}+\mathrm{FP} \times 100 \%$

Accuracy:

Accuracy can measure the overall performance of our proposed soft computing approaches. This can be represented by

Accuracy $(\%)=\mathrm{TP}+\mathrm{TN} / \mathrm{N} \times 100 \%$

\subsection{Comparative Analysis}

In our experiments comparative analysis shall be included with all soft computing approaches which could be used in our proposed work with state-of-the-art technique. Here, we have to compare the detection stage algorithms (SVM \& T2FLS). Before comparing these methods we just produce our results on every stage. At first, we have to produce our pre-processing result, which is displayed in Fig. 7. OD localization and segmentation should provide better results, because without having better results in this stage we cannot extract retinal lesions properly. Snake algorithm could clearly segment the OD. Results of OD localization and segmentation are depicted in Figs. 8 and 9. 


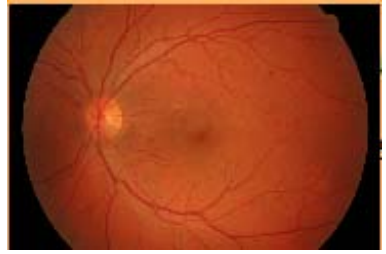

(a)

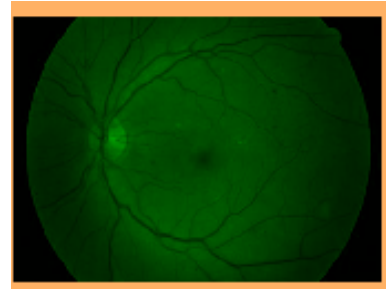

(c)

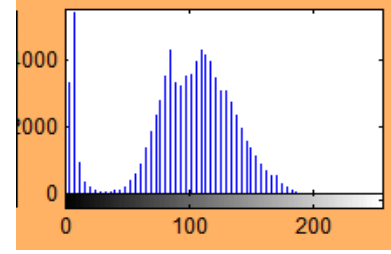

(b)

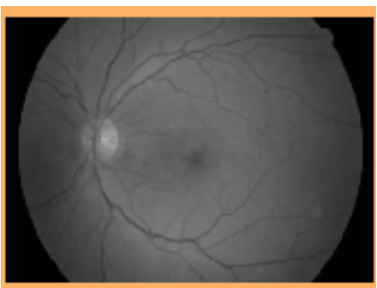

(d)
Fig. 7 Pre-processing results (a) original image (b) contrast enhancement (c) intensity conversion (d) denoising.

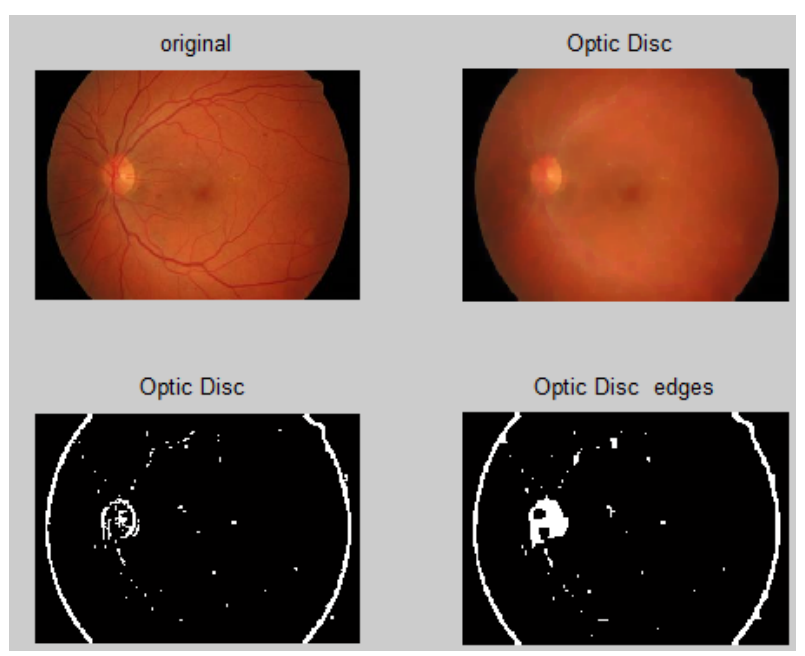

Fig. 8 Optic disc localization.

Blood vessel segmentation could be done with the help of PFCM. This PFCM has been applicable for large datasets and overcome the problem of optimization. Results of PFCM can be depicted in Fig. 10.

Features are extracted based on zero level wavelet decomposition and combined process of co-occurrence matrix. Then the extracted features are selected by ANFIS. Process can be shown in Fig. 11.

Finally, detection of DR stages could be done with the help of T2FLS which could display the results as Mild DR, Moderate DR, Severe DR and PDR. Classifiection comparison has been made with SVM classifier. Table 1 shows the accuracy results for SVM and T2FLS with two databases.

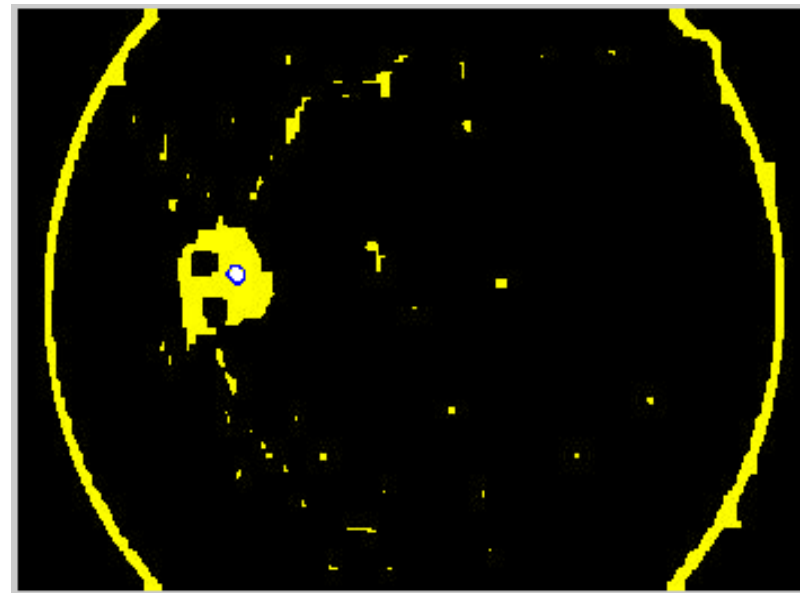

Fig. 9 Optic disc segmentation.

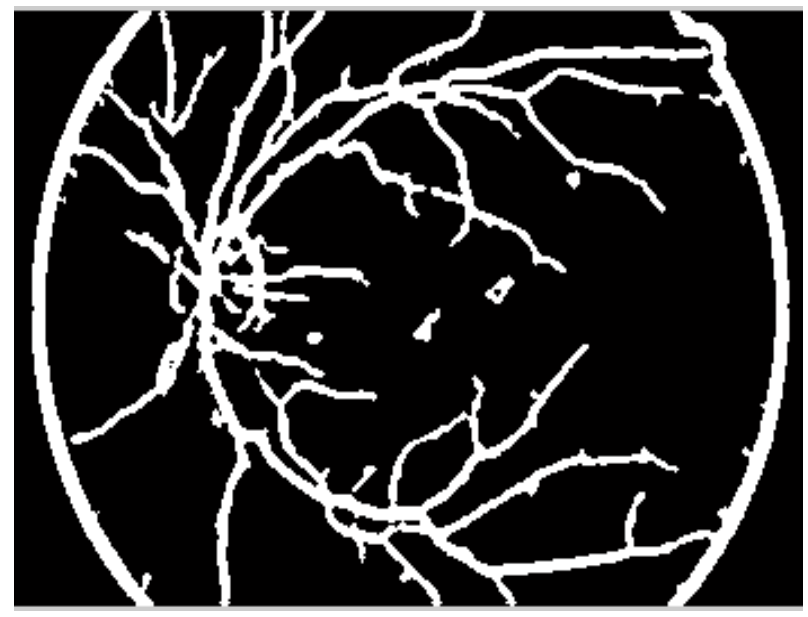

Fig. 10 Blood vessel segmentation.

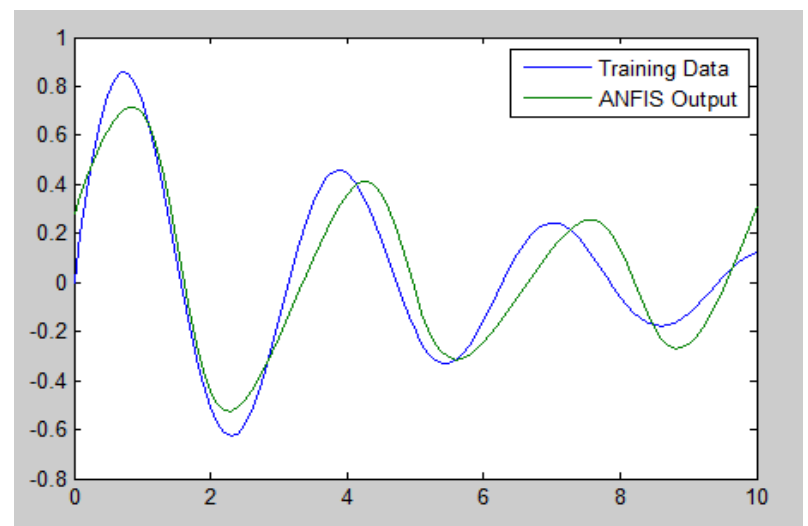

Fig. 11 ANFIS - Feature Selection.

Table 1 Accuracy between SVM and T2FLS.

\begin{tabular}{lllllll}
\hline \multirow{2}{*}{ Database } & \multicolumn{2}{c}{ Sensitivity } & \multicolumn{2}{c}{ Specificity } & \multicolumn{2}{c}{ Accuracy } \\
\cline { 2 - 7 } & SVM & T-2 & \multirow{2}{*}{ SVM } & T-2 & \multirow{2}{*}{ SVM } & T-2 \\
& & FLS & & FLS & & FLS \\
\hline STARE & 86.4 & 87 & 91.7 & 92 & 93.5 & 93.8 \\
DRIVE & 88.9 & 89.2 & 91.2 & 91.7 & 93 & 93.5 \\
\hline
\end{tabular}




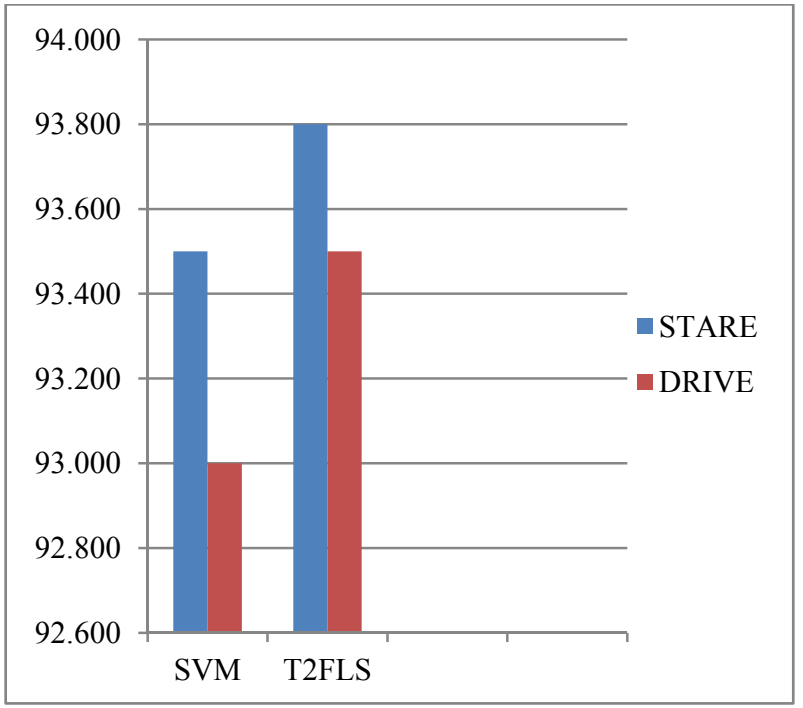

Fig. 12 Performance comparison of SVM and T2FLS.

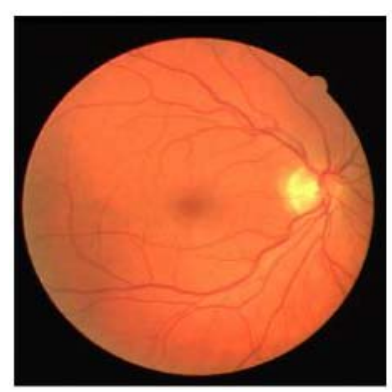

(a)

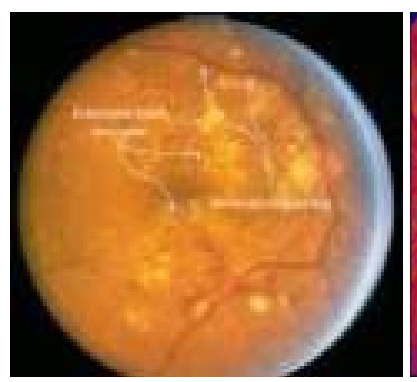

(c)

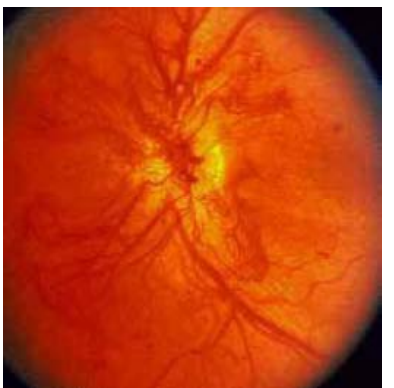

(b)

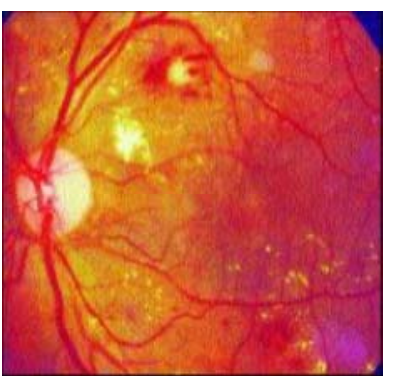

(d)
Fig. 13 Detection of DR stages (a) Mild; (b) Moderate; (c) Severe; (d) PDR.

Accuracy performance can be declared as \%. Comparison results of two databases and two methods are depicted in Fig. 12.

Results of DR stages are classified and also displayed in Fig. 13. Stages are classified as mild, moderate, severe and PDR.

\section{Conclusions}

An automated screening of diabetic retinopathy techniques is discussed in our proposed concept. Soft computing techniques could play an important role in an analysis and detection of diabetic retinopathy. Retinal fundus images are acquired and it can be pre-processed, then optic disc could be localized and segmented. Retinal blood vessel segmentation is done by PFCM. It should clearly segment the blood vessels for clear identification of diabetic retinopathy. Retinal lesion features are extracted based on the combined methodologies of wavelet and co-occurrence matrix. Extraction is fully based on textures. The most important soft computing algorithm is known as ANFIS. It could properly select the features. Finally, detection of diabetic retinopathy could be done by Type-2 Fuzzy Logic System. Classification of several stages of diabetic retinopathy are mild, moderate, severe and PDR. These are classified in an efficient manner. Before classifying these stages we have to classify lesions whether are normal or abnormal. For detection of abnormality we have to perform SVM classification. Our experiments used databases of DRIVE and STARE. These database based input images are processed and also be compared with state-of-the-art techniques. Proposed work should improve the sensitivity, specificity and accuracy, it reaches above 93\%. Soft computing approaches extremely and effectively process and produce accurate results in an easy manner. In future, we will additionally include some combined version of soft computing approaches to improve detection and classification of DR.

\section{References}

[1] Pires, R., Avila, S., Jelinek, F., Wainer, J., and Rocha, A. 2012. "Beyond Lesion-based Diabetic Retinopathy: A Direct Approach for Referral." IEEE Journal of Biomedical and Health Informatics 11 (4): 1-8.

[2] Singh, N., and Tripathi, C. R. 2010. "Automated Early Detection of Diabetic Retinopathy Using Image Analysis Techniques." International Journal of Computer Applications 8 (2): 18-23.

[3] Latare, K. R., and Patil, W. V. 2015. "A Novel Approach for the Detection \& Classification of Diabetic Retinopathy." International Journal on Recent and 
Innovation Trends in Computing and Communication 3 (3): 958-61.

[4] Walvekar, M., and Salunke, G. 2015. "Detection of Diabetic Retinopathy with Feature Extraction Using Image Processing." International Journal of Emerging Technology and Advanced Engineering 5 (1): 133-7.

[5] Ashwin, S., and Kumar, S. A. 2012. "Soft Computing Techniques Based Computer Aided System for Efficient Lung Nodule Detection-A Survey." International Journal of Engineering and Advanced Technology 2 (2): 121-7.

[6] Janakiraman, S., and Gowri, J. 2014. "Robust Color Image Segmentation Using Efficient Soft-Computing Techniques: A survey." American International Journal of Research in Science, Technology, Engineering \& Mathematics 5 (2): 135-9.

[7] EI-Abbadi, N. K., and AI-Saadi, E. H. 2013. "Automatic Detection of Exudates in Retinal Images." International Journal of Computer Science Issues 10 (2): 237-42.

[8] Morales, S., Engan, K., Naranjo, V., and Colomer, A. 2015. "Retinal Disease Screening through Local Binary Patterns." IEEE Journal of Biomedical and Health Informatics. DOI 10.1109/JBHI.2015.2490798.

[9] Roychowdhury, S., Koozekanani, D. D., Kuchinka, S. N., and Parhi, K. K. 2015. "Optic Disc Boundary and Vessel Origin Segmentation of Fundus Images." IEEE Journal of Biomedical and Health Informatics 19 (3): 1118-28.

[10] Sharbaf, M. A., Pourreza, H. R., and Banaee, T. 2015. “A Novel Curvature Based Algorithm for Automatic Grading of Retinal Blood Vessel Tortuosity." IEEE Journal of Biomedical and Health Informatics 20 (2): 586-95.

[11] Gonzalez, A. S., Kaba, D., Li, Y., and Liu, X. 2014. "Segmentation of the Blood Vessels and Optic Disk in Retinal Images." IEEE Journal of Biomedical and Health Informatics 18 (6): 1874-86.

[12] Lazar, I., and Hajdu, A. 2013. "Retinal Microaneurysm Detection through Local Rotating Cross-Section Profile Analysis." IEEE Transaction on Medical Imaging 32 (2): 400-7.

[13] Tang, L., Niemeijer, M., Reinhardt, J. M., Garvin, M. K., and Abramoff, M. D. 2013. "Splat Feature Classification with Application to Retinal Hemorrhage Detection in Fundus Images." IEEE Transaction on Medical Imaging 32 (2): 364-75.

[14] Ganesh, S., and Basha, A. M. 2015. "Automated Detection of Diabetic Retinopathy Using Retinal Optical Images." International Journal of Science, Technology \& Management 4 (2): 136-44.

[15] Maheswari, M. S., and Punnolil, A. 2014. "A Novel Approach for Retinal Lesion Detection Diabetic Retinopathy Images." International Journal of Innovative
Research in Science, Engineering and Technology 3 (3): 1109-14.

[16] Church, J., Chen, Y., and Rice, S. 2008. "A Spatial Median Filter for Noise Removal in Digital Images." In Proceedings of the IEEE Southeast Conference, 618-23.

[17] Prentasic, P. 2006. "Detection of Diabetic Retinopathy in Fundus Photographs." Ph.D. thesis, The Utrecht University Repository.

[18] Mendels, F., Heneghan, C., Harper, P. D., and Reilly, R. B 1999. "Extraction of the Optic Disk Boundary in Digital Fundus Images." In Proceedings of the IEEE Conference on Engineering in Medicine and Biology, doi:10.1109/IEMBS.1999.804304.

[19] Samanta, S., Saha, S. K., and Chanda, B. 2011. "A simple and fast Algorithm to Detect the Fovea Region in Fundus Retinal Image." In Proceedings of the IEEE 2nd International Conference on Emerging Applications of Information Technology, 206-9.

[20] Pal, N. R., Pal, K., Keller, J. M., and Bezdek, J. C. 2005. "A Possibilistic Fuzzy C-Means Clustering Algorithm." IEEE Transaction on Fuzzy Systems 13 (4): 517-30.

[21] Kumari, N., Sharma, B., and Gaur, D. 2012. "Implementation of Possibilistic Fuzzy C-Means Clustering Algorithm in Matlab." International Journal of Scientific \& Engineering Research 3 (11): 1-9.

[22] Manimala, K., and Gokulakrishnan, K. 2014. "Detection of Microaneurysms in Color Fundus Images." International Journal of Emerging Technology and Advanced Engineering 4 (2): 30-7.

[23] Dagher, I., and Taleb, C. 2014. "Image Denoising Using Fourth Order Wiener Filter with Wavelet Quadtree Decomposition." Journal of Electrical and Computer Engineering (March): 1-9.

[24] Islam, B., Ahmed, A., and Kundu, K. 2014. "Texture Feature Based Image Retrieval Algorithms.” International Journal of Engineering and Technical Research 2 (4): 169-73.

[25] Sharma, M., and Mukherjee, S. 2014. "Fuzzy C-Means, ANFIS and Genetic Algorithm for Segmenting Astrocytoma-A Type of Brain Tumor." International Journal of Artificial Intelligence 3 (1): 16-23.

[26] Zarandi, M. H. F., Nejad, F. M., and Zakeri, H. 2012. "A Type-2 Fuzzy Model Based on Three Dimensional Membership Functions for Smart Thresholding in Control Systems." In Fuzzy Controllers-Recent Advances in Theory and Applications, edited by Iqbal, S., Boumella, N., and Garcia, J. C. F. Vienna: InTechOpen.

[27] Hosseini, R., Qanadli, S. D., Barman, S., Mazinani, M., Ellis, T., and Dehmeshki, J. 2012. "An Automatic Approach for Learning and Tuning Gaussian Interval Type-2 Fuzzy Membership Functions Applied to Lung CAD Classification System.” IEEE Transactions on Fuzzy 
Systems 20 (2): 224-34.

[28] Jitpakdee, P., Aimmanee, P., and Uyyanonvara, B. 2012. "A Survey on Hemorrhage Detection in Diabetic Retinopathy Retinal Images." In Proceedings of the IEEE 9th Conference on Electrical Engineering/Electronics, Computer, Telecommunications and Information Technology, 1-4.

[29] Paranjpe, M. J., and Kakatkar, M. N. 2014. "Review of
Methods for Diabetic Retinopathy Detection and Severity Classification." International Journal of Research in Engineering and Technology 3 (3): 619-24.

[30] Tamilarasi, M., and Duraiswamy, K. 2014. “A Survey for Automatic Detection of Non-proliferative Diabetic Retinopathy." International Journal of Innovative Research in Computer and Communication Engineering 2 (1): 2418-22. 